\title{
Elaboração de um conjunto de métricas de qualidade para avaliação de experiências de aprendizagem com realidade aumentada
}

\author{
Julio A.R. Carraro ${ }^{1}$, Fabiola A.D. Spredemann ${ }^{1}$, Eliane Pozzebon ${ }^{1}$ \\ Josete Mazon ${ }^{1}$, Iane F. Sousa ${ }^{1}$, Alexandre M. Costa ${ }^{2}$ \\ ${ }^{1}$ Laboratório de Tecnologias Computacionais - Universidade Federal de Santa Catarina \\ 88.906-072 - Araranguá - SC - Brasil \\ ${ }^{2}$ Departamento de Ciências da Administração - Universidade Federal de Santa Catarina \\ 88.040-900 - Florianópolis - SC - Brasil \\ \{julio.a.carraro, fabispredemann, epozzebon, jomazoncmf, ianefran\} @gmail.com \\ alexandre.marinodufsc.br
}

\begin{abstract}
This article aims to present the development of a set of metrics for the quality evaluation of augmented reality applications related to education. Using the Goal Question Metric method, 8 classes of metrics were developed and subsequently applied to an augmented reality development project. The considered metrics were effective in mapping problems in the development process, facilitating the correction and adaptation procedures. Consequently, the use of appropriate metrics enabled a greater control over the quality of applications, generating compatible experiences with their objectives.
\end{abstract}

Resumo. O presente artigo objetiva apresentar a elaboração de um conjunto de métricas para a avaliação da qualidade das aplicações de realidade aumentada relacionadas à educação. A partir do método Goal Question Metric, foram desenvolvidas 8 classes de métricas e posteriormente aplicadas em um projeto de desenvolvimento de realidade aumentada. As métricas consideradas foram efetivas no mapeamento de problemas no processo de desenvolvimento, facilitando assim os procedimentos de correção e adaptação. Consequentemente, a utilização de métricas adequadas possibilitou um maior controle da qualidade das aplicações, gerando experiências compatíveis com seus objetivos.

\section{Introdução}

O avanço na tecnologia é cada vez mais presente na educação. O uso das tecnologias tem possibilitado um aprendizado mais significativo, modificando a forma como a construção do conhecimento é compartilhada entre professores e alunos na sala de aula [Lopes et al. 2019]. A realidade aumentada (RA) se enquadra dentro destas novas tecnologias educacionais, combinando elementos reais e virtuais no ambiente, de maneira interativa e em tempo real [Azuma 1997]. A implementação de experiências de aprendizado com realidade aumentada (EARAs) como método enriquecedor no processo ensino aprendizagem tem sido ampla em ambientes educacionais, principalmente através de dispositivos móveis [Becker et al. 2018] [Lopes et al. 2019]. As EARAs são caracterizadas 
IX Congresso Brasileiro de Informática na Educação (CBIE 2020)

Anais do XXXI Simpósio Brasileiro de Informática na Educação (SBIE 2020)

como aplicações de RA que atuam no processo ensino aprendizagem de forma a facilitar a experiência dos envolvidos [Santos et al. 2013].

Intuindo a qualidade e construção eficiente de software, é essencial agregar técnicas e métodos que sejam adequados a todos os processos de desenvolvimento. Entretanto, o conceito de qualidade de software acaba por ser complexo e muitas vezes subjetivo, sendo que a adoção da definição de qualidade costumeira a produtos palpáveis não pode ser aplicada sem inconsistência. A qualidade de software está intrinsecamente vinculada a existência de atividades de garantia, planejamento e controle, por meio de métricas de análise e padrões de desenvolvimento. Nesse sentido, uma métrica pode ser caracterizada como um tipo de medição que se refira a um sistema, processo ou documentação de software [Schulmeyer 1992].

É evidente a alta densidade de técnicas de avaliação de software dentro de um âmbito geral, todavia, a análise da qualidade de aplicações RA carece de uma ampla e detalhada cobertura literária. De acordo com a revisão sistemática elaborada por Dunser (2008), apenas $8 \%$ das publicações que tratam de aplicações em RA apresentam algum tipo de avaliação acerca do desenvolvimento [Dey et al. 2018] [Dünser et al. 2008] .

Tendo em vista o referido até então, tem-se como propósito elaborar um conjunto de métricas para análise de qualidade de aplicações de RA relacionadas ao âmbito pedagógico. Ansiando a sua validação, os conjuntos desenvolvidos serão aplicados às etapas de desenvolvimento de um projeto de RA elaborado no Laboratório de Tecnologias Computacionais (LabTeC) da Universidade Federal de Santa Catarina - Campus Araranguá. Ademais, com base nas métricas escolhidas, é pretendido nortear e servir de embasamento para outros pesquisadores que desejam iniciar o desenvolvimento de artefatos de RA correlacionados à educação.

O presente trabalho está estruturado em seis seções, da seguinte maneira: o conteúdo introdutório referente a pesquisa, situado na presente seção. Na seção 2 são apresentados os trabalhos correlatos e fundamentação teórica. A seção 3 descreve plenamente o desenvolvimento do projeto. A seção 4 aborda sobre a metodologia desenvolvida para aplicação. A análise e discussão dos resultados obtidos está descrita na seção 5. Por fim, as considerações finais são apresentadas na sexta seção.

\section{Trabalhos correlatos e fundamentação teórica}

É relevante a breve apresentação e discussão do material literário resultante de pesquisas relacionadas com o presente estudo e que contribuem com o objetivo do mesmo. As pesquisas abaixo denotam técnicas para elaboração de métricas de software, ferramentas para análise da qualidade de aplicações em RA e considerações quanto ao desenvolvimento de artefatos educacionais de RA.

Em relação à elaboração de métricas de software, a pesquisa de Gomes (2011) objetiva estabelecer metodologias para a escolha de conjuntos de métricas com base no objeto a ser desenvolvido [Gomes 2011]. Nessa mesma linha, Madureira et al. (2017) exibem uma ampla revisão literária da área, elencando metodologias como Goal Qustion Metric, Goal-Driven Software Measurement e Practical Software Measurement, as aplicando em um estudo de caso [Madureira et al. 2017]. Os autores concluem que a seleção de um método de análise que considera a natureza da aplicação é fundamental para a 
IX Congresso Brasileiro de Informática na Educação (CBIE 2020)

Anais do XXXI Simpósio Brasileiro de Informática na Educação (SBIE 2020)

manutenção da qualidade do processo de desenvolvimento e do software final.

Samini e Palmerius (2017) e Bowman, Johnson e Hodges (2001) buscam coletar quais são as principais métricas de avaliação utilizadas em projetos de RA [Samini 2017] [Bowman et al. 2001]. Baseados na análise literária, os autores elencam uma série de métricas consideradas essenciais para o desenvolvimento de ambientes virtuais de qualidade. As métricas catalogadas demonstram principalmente a existência de preocupações sobre a usabilidade das aplicações, como o comportamento do objeto tridimensional perante as ações do usuário.

A pesquisa de Santos et al. (2013) investiga EARAs relacionados à educação de aprendizes do ensino fundamental e médio, efetivando uma análise qualitativa das características técnicas das aplicações [Santos et al. 2013]. Os pesquisadores categorizam aspectos intrínsecos para softwares educacionais de RA com base em teorias pedagógicas de aprendizagem multimídia, aprendizagem experimental e visão animada. Os aspectos levantados foram também fundamentados a partir das características comuns dos ambientes averiguados.

Apesar das considerações sobre métodos de análise de qualidade de software e características essenciais em aplicações de RA, nenhum dos trabalhos de pesquisa analisados apresentaram propostas específicas para a construção de um conjunto de métricas de avaliação para o desenvolvimento de EARAs. Um dos diferenciais da presente pesquisa é justamente a elaboração de um método de análise adequado e que leve em consideração os fundamentos de qualidade de software e aspectos pedagógicos necessários.

Tendo em vista o objetivo do presente trabalho, é relevante discorrer acerca de métricas de qualidade de software. Um sistema de métricas objetiva a correlação de medidas individuais almejando coletar dados acerca da eficácia da entidade que sofre a análise [Pressman 2016]. Sendo assim, as métricas são medidas qualitativas do grau em que algum processo do desenvolvimento geral do projeto apresentam características [Schulmeyer 1992]. Além do citado, é possível elencar três principais propriedades das métricas de avaliação de projetos de software, segundo Theden (2013):

- Informação: métricas possibilitam a coleta de dados importantes ao longo do desenvolvimento do projeto;

- Quantificação de dados: todos os dados coletados e suas devidas relações com as etapas de desenvolvimento são mensuráveis e classificáveis;

- Coleção de informações: a partir da construção de uma coleção de métricas, processos complexos podem ser organizados e representados de maneira simplificada.

Ademais das características fundamentais das métricas de avaliação, a sua aplicação pode ser dada em diferentes componentes do desenvolvimento de software, tanto no escopo de processos, quanto no produto em si [Theden 2013].

\section{Desenvolvimento do projeto de RA}

Antes de estabelecer a metodologia, é necessário compreender a dinâmica do processo e as etapas de um projeto de desenvolvimento de EARAs. O projeto de desenvolvimento das aplicações em RA na qual a metodologia foi aplicada é parte integrante do projeto de extensão RA Escolas, desenvolvido pelo LabTeC. A equipe de desenvolvimento é responsável por produzir inúmeros artefatos que são aplicados em escolas públicas por meio de oficinas e distribuídos num repositório online. 
O objetivo do projeto é a construção e aplicação de experiências de RA para auxiliar o ensino de ciências para alunos do ensino fundamental e médio. A interação com os modelos tridimensionais ocorre a partir do acesso da aplicação com um dispositivo móvel e marcadores de acesso.

A equipe de desenvolvimento é composta por pesquisadores, docentes e discentes dos cursos de bacharelado em Medicina e em Engenharia de Computação da universidade. A seguir, as etapas de desenvolvimento das experiências serão introduzidas e relatadas.

\subsection{Pesquisa e validação científica}

Os responsáveis pelo arcabouço teórico são incumbidos pela seleção da bibliografia base para consulta e elaboração do material fundamental. O escopo da bibliografia é analisado e respeitado, com a finalidade de manter a complexidade adequada para os níveis de educação do projeto. A partir da coleta de dados de acordo com a literatura recomendada, os modelos são discutidos e explanados. Esboços dos modelos são desenvolvidos de maneira a exemplificar o conteúdo que deverá ser considerado na etapa de modelagem tridimensional. Além dos esboços, materiais textuais explicativos também são produzidos.

\subsection{Modelagem tridimensional}

A partir do material teórico desenvolvido na primeira etapa, o conteúdo é analisado com cautela antes de iniciar a construção do modelo. O objetivo da modelagem é reproduzir o modelo decidido na etapa de pesquisa e validação científica, em um objeto tridimensional, preservando as características essenciais para a adequada aplicação no ensino.

\subsection{Texturização gráfica}

Finalizada a etapa de modelagem, inicia-se a texturização gráfica. Nesta etapa, o modelo tridimensional recebe as texturas e materiais gráficos, de acordo com o que foi definido na primeira etapa, priorizando a representação realística das texturas reais.

\subsection{Animação}

Até a presente etapa o objeto tridimensional é estático, contudo, o mesmo ainda precisa ser animado de modo a representar de forma mais verossímil o comportamento do cenário em questão. Finalizada a animação, as texturas criadas na terceira etapa são adicionadas ao modelo.

\subsection{Design Gráfico}

Nesta etapa são produzidas as telas gráficas e uma interface de usuário polida e responsiva. Ademais, é criado o material gráfico onde os marcadores de RA serão anexados.

\subsection{Programação do ambiente tridimensional}

A partir das aplicações das texturas, o modelo tridimensional está completo. Contudo, recursos de interatividade dinâmica ainda não foram agregados à aplicação. A presente etapa tem como objetivo programar o ambiente, com o propósito de possibilitar que os aprendizes consigam interagir com o objeto tridimensional. As animações criadas na etapa 3.4 são programadas nesta etapa. Após a finalização das atividades de programação necessárias, o modelo é renderizado, gerando um marcador para o devido acesso por dispositivo móvel. 
IX Congresso Brasileiro de Informática na Educação (CBIE 2020)

Anais do XXXI Simpósio Brasileiro de Informática na Educação (SBIE 2020)

\subsection{Testes finais e validação técnica}

Por último, temos a etapa de testes finais e validação técnica, objetivando a aplicação de testes ao ambiente desenvolvido. Técnicas de retroação são acionadas caso algum erro, falha, ou anomalia ocorra nas etapas.

\section{Metodologia}

Um único projeto de desenvolvimento pode abrigar um conjunto vasto de métricas, de modo a tornar a verificação da qualidade um trabalho custoso e complexo. Levando tal fato em consideração, é necessário mapear corretamente as métricas de avaliação de forma a contribuir para o compreendimento dos processos de desenvolvimento sem um aumento desnecessário de esforço e complexidade de avaliação.

\subsection{Goal Question Metric}

A fim de alcançar um conjunto sólido de métricas, foi aderido ao projeto a abordagem Goal Question Metric (GQM), um sistema de avaliação direcionado aos objetivos amplamente utilizado por equipes de produção de software [Chagas 2019]. O método proposto por Basili e Caldiera [Caldiera 1994] é estruturado em três níveis: conceitual (lida com os objetivos definidos a partir de uma entidade), operacional (trata do conjunto de perguntas que levarão um determinado objetivo a ser alcançado) e quantitativo (os questionamentos são respondidas a partir de métricas associadas a cada pergunta estabelecida anteriormente). O método foi escolhido devido ao seu maduro e desenvolvido mecanismo de avaliação para projetos com objetivos bem definidos.

\subsection{Constituição metodológica}

Para o planejamento dos objetivos, as ponderações de [Santos et al. 2013] sobre a construção de EARAs foram consideradas, sendo estas: registro no mundo real, visualização contextual e visualização háptica. A primeira consideração lida com informações que devem ser exibidas em objetos físicos reais, como a própria projeção tridimensional da aplicação. A visualização contextual é considerada para garantir a congruência da aplicação com o objeto de aprendizado, tendo relação com a usabilidade do ambiente. A última definição trata da manipulação do objeto virtual a partir de ações motoras do usuário (rotação, translação, manipulação dimensional, entre outras).

Outra consideração importante para os objetivos é a integração da aplicação de RA em salas de aula. De modo a integrar efetivamente os EARAs no ambiente escolar, é imprescindível que o tutor direcione o conteúdo juntamente com o recurso tecnológico, de modo a apoiar o processo ensino-aprendizagem [Dexter 2002]. Destarte, é necessário que a aplicação forneça mecanismos que propiciem o trabalho do tutor e garantam uma experiência aprazível.

Considerando as preocupações discutidas até então e as considerações sobre análise de software, é possível elencar os objetivos gerais para o desenvolvimento de EARAs:

1. As aplicações precisam ser estáveis na maioria dos dispositivos, levando em conta a diversidade de configurações de hardware dos mesmos;

2. As aplicações devem apresentar os princípios de registro no mundo real, visualização conceitual e háptica; 
3. As aplicações devem conter recursos que auxiliem o tutor em sala de aula.

Estando os objetivos gerais de desenvolvimento esclarecidos, é possível definir os questionamentos, as classes das métricas e métricas específicas que serão utilizadas. Oito classes de métricas foram estabelecidos a partir da definição dos objetivos, de modo a contemplar as etapas de desenvolvimento. Métricas específicas foram elaboradas para responder os questionamentos. A Tabela 1 exibe as considerações do método GQM para o projeto.

Tabela 1. Questões e métricas elaboradas em relação aos objetivos

\begin{tabular}{|c|c|c|c|}
\hline $\begin{array}{l}\text { Objetivo } \\
\text { (OB) }\end{array}$ & Questões (Q) & Métricas (M) & Classificação \\
\hline \multirow[t]{2}{*}{1} & $\begin{array}{l}\text { 1.1) Qual é a quantidade total de dados produzidos em } \\
\text { cada etapa do desenvolvimento e o tamanho total do } \\
\text { projeto? }\end{array}$ & $\begin{array}{l}\text { 1.1.a) Constatação do tamanho } \\
\text { computacional ideal e real dos } \\
\text { arquivos produzidos em cada } \\
\text { aplicação. }\end{array}$ & $\begin{array}{l}\text { Tamanho do } \\
\text { sistema }\end{array}$ \\
\hline & $\begin{array}{l}\text { 1.2) Qual a quantidade dos polígonos dos modelos } \\
\text { tridimensionais? }\end{array}$ & $\begin{array}{l}\text { 1.2.a) Constatação ideal e real dos } \\
\text { vértices, arestas e faces de cada } \\
\text { modelo tridimensional. }\end{array}$ & $\begin{array}{l}\text { Complexidade } \\
\text { geométrica }\end{array}$ \\
\hline \multirow[t]{4}{*}{2} & $\begin{array}{l}\text { 2.1) Qual o tamanho de resolução dos arquivos de } \\
\text { texturização gráfica? }\end{array}$ & $\begin{array}{l}\text { 2.1.a) Constatação ideal e real da } \\
\text { resolução em pixels dos } \\
\text { documentos de textura. }\end{array}$ & $\begin{array}{l}\text { Complexidade } \\
\text { gráfica }\end{array}$ \\
\hline & $\begin{array}{l}\text { 2.2) Quais são os fatores de manipulação (e seus graus } \\
\text { de liberdade) são presentes nas aplicações? }\end{array}$ & $\begin{array}{l}\text { 2.2.a) Constatação dos fatores de } \\
\text { manipulação e dos seus graus de } \\
\text { liberdade. }\end{array}$ & $\begin{array}{l}\text { Complexidade } \\
\text { de } \\
\text { manipulação }\end{array}$ \\
\hline & \multirow[t]{2}{*}{$\begin{array}{l}\text { 2.3) Quanto tempo os recursos da aplicação levam para } \\
\text { serem executados a partir do comando do usuário? }\end{array}$} & $\begin{array}{l}\text { 2.3.a) Marcação do tempo } \mathrm{de} \\
\text { resposta das ações de manipulação. }\end{array}$ & \multirow[t]{2}{*}{$\begin{array}{l}\text { Tempo de } \\
\text { conclusão }\end{array}$} \\
\hline & & $\begin{array}{l}\text { 2.3.b) Marcação do tempo de } \\
\text { resposta das ações de acesso } \\
\text { (botões, telas, etc). }\end{array}$ & \\
\hline \multirow[t]{3}{*}{3} & $\begin{array}{l}\text { 3.1) Quais são os recursos que oferecem um } \\
\text { complemento teórico à aplicação? }\end{array}$ & $\begin{array}{l}\text { 3.1.a) Averiguação dos recursos } \\
\text { gráficos de complementação } \\
\text { teórica. }\end{array}$ & $\begin{array}{l}\text { Conteúdo } \\
\text { auxiliar }\end{array}$ \\
\hline & $\begin{array}{l}\text { 3.2) Quais são os recursos que atuam no auxílio da } \\
\text { avaliação do aprendiz? }\end{array}$ & $\begin{array}{l}\text { 3.2.b) Averiguação dos recursos } \\
\text { gráficos de avaliação. }\end{array}$ & $\begin{array}{l}\text { Conteúdo } \\
\text { avaliativo }\end{array}$ \\
\hline & $\begin{array}{l}\text { 3.3) Quais são os recursos que atuam na instrução de uso } \\
\text { da aplicação? }\end{array}$ & $\begin{array}{l}\text { 3.3.c) Averiguação dos recursos } \\
\text { gráficos instrucionais. }\end{array}$ & $\begin{array}{l}\text { Conteúdo } \\
\text { instrucional }\end{array}$ \\
\hline
\end{tabular}

Considerando que o trabalho pretende construir um conjunto de métricas gerais para a avaliação de qualidade de EARAs, métricas de tempo e produtividade não foram incluídas no conjunto elaborado devido a particularidade de cada equipe de desenvolvimento.

\section{Análise e discussão dos resultados}

O conjunto de métricas estabelecidas previamente foi empregado ao processo de desenvolvimento das aplicações de RA. O período de desenvolvimento compreendido entre junho e dezembro de 2019 foi designado para a aplicação do conjunto de métricas de qualidade, no qual foram elaborados 14 aplicações de RA abordando conteúdos a respeito de células biológicas. Em seguida, serão evidenciados os resultados da análise com base nos objetivos, questionamentos e métricas consolidados a partir do método GQM. 
IX Congresso Brasileiro de Informática na Educação (CBIE 2020)

Anais do XXXI Simpósio Brasileiro de Informática na Educação (SBIE 2020)

\subsection{OB1}

Em relação ao objetivo de estabilidade do sistema, duas questões foram produzidas com uma métrica especificada para cada questionamento. O questionamento Q1.1 lida com o tamanho computacional dos dados de cada aplicação, sendo que a métrica M1.1.a foi aplicada para tal constatação. Testes foram realizados com o objetivo de mapear o tamanho adequado que a aplicação deve conter sem que ocorram instabilidades e travamentos durante a sua execução. Uma máquina virtual (MV) que emula as características de hardware de um dispositivo móvel de baixa capacidade foi configurada para a efetivação de testes de performance. Segundo os resultados dos testes, a aplicação apresenta um comportamento estável com um tamanho absoluto de até 5 megabytes (MB). Analisando os arquivos, todas as aplicações apresentavam um tamanho superior ao limite apontado, sendo assim, técnicas de compressão de dados foram aplicadas aos arquivos das aplicações de modo a garantir um tamanho inferior ao limite.

A complexidade geométrica do objeto tridimensional é outro fator que influencia a fluidez de execução da aplicação em RA. A métrica M1.2.a foi empregada para atender a questão Q1.2. Os testes executados na MV indicaram que a aplicação exibe um comportamento consistente com objetos de complexidade poligonal de até 100.000 polígonos. Grande parte dos modelos produzidos apresentavam uma cardinalidade poligonal que excedia o limite constatado. Para alcançar a complexidade adequada, processos de retopologia e compressão geométrica foram utilizados nos modelos, reduzindo consideravelmente o número de polígonos.

A Tabela 2 apresenta os dados coletados com o auxílio das métricas de qualidade.

Tabela 2. Dados coletados a partir da aplicação das métricas

\begin{tabular}{|c|c|c|c|c|c|c|c|c|c|c|c|c|c|c|}
\hline $\begin{array}{c}\text { Aplicação } \\
\text { (AP) }\end{array}$ & AP1 & AP2 & AP3 & AP4 & AP5 & AP6 & AP7 & AP8 & AP9 & AP10 & AP11 & AP12 & AP13 & AP14 \\
\hline $\begin{array}{c}\text { Tamanho } \\
\text { antes da } \\
\text { otimização }\end{array}$ & $6,8 \mathrm{MB}$ & 9,1MB & $7,2 \mathrm{MB}$ & $8.5 \mathrm{MB}$ & $7,3 \mathrm{MB}$ & $5.2 \mathrm{MB}$ & $5.7 \mathrm{MB}$ & 6.1MB & $5.3 \mathrm{MB}$ & $6.3 \mathrm{MB}$ & $8.6 \mathrm{MB}$ & $5.4 \mathrm{MB}$ & $6.0 \mathrm{MB}$ & $8.9 \mathrm{MB}$ \\
\hline $\begin{array}{c}\text { Tamanho após } \\
\text { otimização }\end{array}$ & $3,2 \mathrm{MB}$ & $4,6 \mathrm{MB}$ & $2,9 \mathrm{MB}$ & $4,5 \mathrm{MB}$ & $4,7 \mathrm{MB}$ & $4,2 \mathrm{MB}$ & $4,2 \mathrm{MB}$ & $3,8 \mathrm{MB}$ & $4,1 \mathrm{MB}$ & $3,8 \mathrm{MB}$ & $4.3 \mathrm{MB}$ & $2.8 \mathrm{MB}$ & $3.6 \mathrm{MB}$ & $4.4 \mathrm{MB}$ \\
\hline $\begin{array}{c}\text { Polígonos } \\
\text { antes da } \\
\text { otimização }\end{array}$ & 203.107 & 217.196 & 189.744 & 241.020 & 239.266 & 287.154 & 201.003 & 292.145 & 301.241 & 294.442 & 293.120 & 295.904 & 293.855 & 298.712 \\
\hline $\begin{array}{c}\text { Polígonos após } \\
\text { otimização }\end{array}$ & 53.247 & 67.887 & 64.102 & 69.820 & 58.433 & 72.441 & 65.963 & 71.009 & 86.523 & 81.847 & 74.213 & 77.280 & 73.965 & 80.852 \\
\hline
\end{tabular}

\subsection{OB2}

Para satisfazer o objetivo OB2, três questões e quatro métricas foram construídas e consideradas. A questão Q2.1 é relacionada com a preocupação do nível de detalhes que o modelo tridimensional expõe em comparação com o modelo real utilizado de base. A resolução das texturas interfere diretamente na verossimilhança e visualização conceitual dos modelos produzidos. Além da representação visual, é necessário que os arquivos de textura se adequem ao limite de tamanho estabelecido de modo a cumprir também com o objetivo OB1. A partir de uma resolução com 1.048 .576 pixels, as texturas representam os modelos reais de forma adequada, contudo, os testes de estabilidades conduzidos na MV apontaram que texturas com resolução superior a 4.194 .304 pixels tornam a experiência mais lenta. Em consonância com os testes, todas as texturas das aplicações foram padronizadas com uma resolução de 1024 pixels quadrados.

O questionamento Q2.2 e a métrica M2.2.a foram organizados para garantir o conceito de visualização háptica e registro no mundo real pretendido no objetivo OB2. Foram 
desenvolvidos quatro fatores de manipulação: rotação, translação, transposição e escala. Os fatores de rotação, translação e escala apresentam três graus de liberdade, enquanto o fator de transposição apresenta dois graus de liberdade. Os fatores em conjunto com seus graus de liberdade possibilitam uma manipulação plena do objeto em RA, além de permitir que as manipulações do usuário fiquem registradas em tempo real na aplicação.

Para a questão Q2.3, duas métricas foram elaboradas. A métrica M2.3.a foi direcionada para a análise do tempo de execução das ações de manipulação, enquanto a métrica M2.3.b foi destinada para a verificação do tempo de execução dos botões, gatilhos e demais funções de acesso das aplicações. É importante para a usabilidade do ambiente que o tempo de resposta para as ações de interação do usuário tenha uma curta duração, sendo que o intervalo ideal varia de 0,10 até 1 segundos(s) [Nielsen 1994]. Testes executados na MV evidenciaram que o tempo de resposta das funções de manipulação e acesso apresentaram valores adequados, entretanto, melhorias nas matrizes de manipulação foram implementadas para alcançar um tempo ainda menor, como pode ser averiguado na Tabela 3. Vale ressaltar que o tempo de resposta constatado é válido para todas as aplicações desenvolvidas, já que o código das funções foi reutilizado.

Tabela 3. Dados coletados a partir da aplicação das métricas

\begin{tabular}{|c|c|c|c|c|c|}
\hline Função & Rotação & Translação & Transposição & Escala & Acesso \\
\hline Tempo antes da otimização (s) & 0,26 & 0,33 & 0,19 & 0,17 & 0,19 \\
\hline Tempo após otimização (s) & 0,15 & 0,21 & 0,11 & 0,10 & 0,10 \\
\hline
\end{tabular}

\subsection{OB3}

Finalmente, três questões e três métricas foram ponderadas em relação ao objetivo OB3. Os questionamentos e métricas visam mapear os recursos gráficos implementados que servem de subsídio para o tutor que utiliza o ambiente com seus aprendizes. As métricas evidenciaram a ausência destes recursos gráficos, o que mobilizou a implementação dos recursos pela equipe de desenvolvimento. Relacionada ao questionamento Q3.1, a métrica M3.1.a foi utilizada para verificar os recursos teóricos complementares nas aplicações. Foram desenvolvidos pontos de interesse no modelo tridimensional das aplicações, que a partir do toque, expõem informações específicas sobre o componente celular.

A respeito da questão Q3.2, a métrica M3.2.a foi adotada para a análise dos recursos de avaliação das experiências. Questionários objetivos que abordam a temática do modelo celular apresentado foram desenvolvidos para as aplicações.

Enfim, a questão e métrica finais foram tomadas para verificar recursos que oferecem instruções de interação com os modelos. Quando o usuário acessa a experiência pela primeira vez com o dispositivo móvel, a aplicação exibe um tutorial animado que exemplifica como o modelo deve ser manipulado. A Figura 1 demonstra, nesta ordem, os pontos de interesse, o conteúdo de um dos pontos, o questionário e o conteúdo tutorial.

\section{Considerações finais}

Este artigo apresentou um conjunto de métricas para avaliação da qualidade de EARAs fundamentado em aspectos pedagógicos e de engenharia de software. Assim, para atingir 


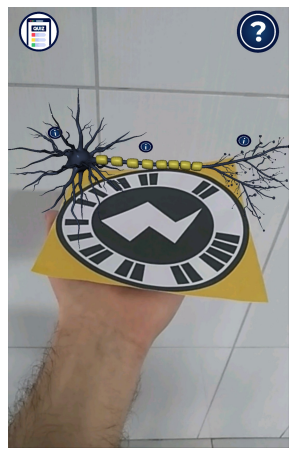

(a)

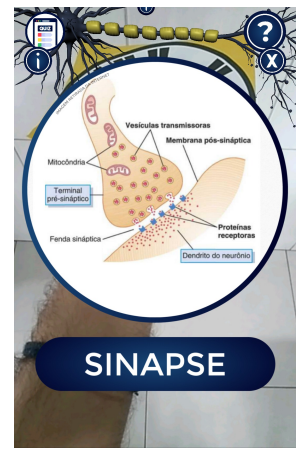

(b)

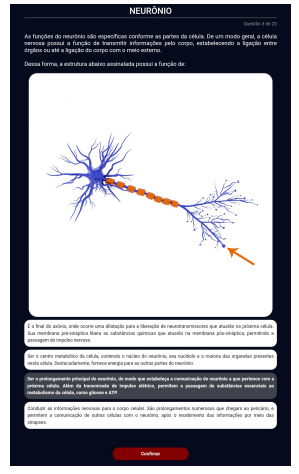

(c)

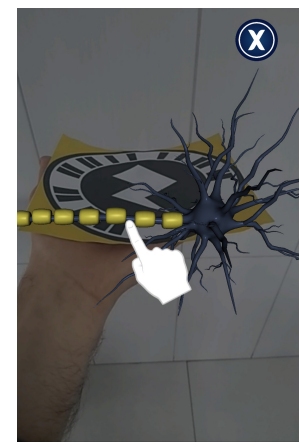

(d)

Figura 1. Recursos gráficos auxiliares

o objetivo deste trabalho, foi optado pela implementação do método GQM como instrumento de avaliação. A validação metodológica foi realizada a partir da aplicação dos objetivos, questionamentos e métricas no processo de desenvolvimento do projeto de RA executado em um laboratório de tecnologia da universidade.

Com base nas oito classes de métricas e nas métricas específicas elaboradas a partir dos questionamentos, dados do desenvolvimento das experiências foram coletados e analisados. Testes foram efetuados em MV em relação aos questionamentos de cada objetivo, de modo a obter valores consideráveis para garantir a estabilidade do ambiente. A partir da análise dos resultados adquiridos com o auxílio das métricas, foi observado que alguns componentes do desenvolvimento apresentavam comportamentos dissonantes dos objetivos elencados, sendo especificamente problemas de estabilidade e falta de material auxiliar. Após a análise dos resultados, processos de correção e otimização foram conduzidos para retificar os componentes problemáticos, e consequentemente, auxiliar no cumprimento dos objetivos pautados.

O método GQM se mostrou satisfatório para a construção de objetivos adequados para um ambiente de RA, permitindo a construção de um conjunto de métricas sólidas para avaliação da qualidade dos artefatos concebidos. Pode-se concluir que o objetivo do artigo foi alcançado, visto que foi possível a constituição de um grupo de métricas gerais capaz de rastrear as áreas problemáticas do desenvolvimento e permitir as devidas correções, assim, auxiliando na manutenção de qualidade do projeto. Assim sendo, o método de análise elaborado na presente pesquisa tem o potencial de basear a construção qualificada de artefatos de RA relacionados à educação.

Como trabalhos futuros, é almejado a aplicação das classes de métricas em estudos de caso com outras equipes de desenvolvimento de EARAs, de modo a obter novos dados para validação e adequação das métricas.

\section{Referências}

Azuma, R. T. (1997). Uma pesquisa sobre realidade aumentada. Presença: Teleoperadores e Ambientes Virtuais, 6(4):355-385.

Becker, S. A., Brown, M., Dahlstrom, E., Davis, A., DePaul, K., Diaz, V., and Pomerantz, J. (2018). Nmc horizon report: 2018 higher education edition. Louisville, CO: 
IX Congresso Brasileiro de Informática na Educação (CBIE 2020)

Anais do XXXI Simpósio Brasileiro de Informática na Educação (SBIE 2020)

\section{EDUCAUSE.}

Bowman, D. A., Johnson, D. B., and Hodges, L. F. (2001). Testbed evaluation of virtual environment interaction techniques. Presence: Teleoperators \& Virtual Environments, 10(1):75-95.

Caldiera, Victor R Basili1 Gianluigi e Rombach, H. D. (1994). The goal question metric approach. Encyclopedia of software engineering, pages 528-532.

Chagas, Jonas M e da Silva, J. P. S. (2019). Um plano goal/question/metrics para aprendizado baseado em problemas na engenharia de software. In Anais da III Escola Regional de Engenharia de Software, pages 73-80. SBC.

Dexter, S. (2002). etips-educational technology integration and implementation principles. In Designing instruction for technology-enhanced learning, pages 56-70. IGI Global.

Dey, A., Billinghurst, M., Lindeman, R. W., and Swan, J. (2018). A systematic review of 10 years of augmented reality usability studies: 2005 to 2014. Frontiers in Robotics and $A I, 5: 37$.

Dünser, A., Grasset, R., and Billinghurst, M. (2008). A survey of evaluation techniques used in augmented reality studies. Human Interface Technology Laboratory New Zealand.

Gomes, M. M. P. (2011). Métricas e medição no processo de desenvolvimento de software: estudo de caso. $\mathrm{PhD}$ thesis, Instituto Universitário de Lisboa.

Lopes, L. M. D., Vidotto, K. N. S., Pozzebon, E., and Ferenhof, H. A. (2019). Inovações educacionais com o uso da realidade aumentada: Uma revisão sistemática. Educação em Revista, 35.

Madureira, J. S. et al. (2017). Avaliação de equipes de desenvolvimento de software por meio de métricas orientadas a objeto. Master's thesis, Universidade Federal de Sergipe.

Nielsen, J. (1994). Usability engineering. Morgan Kaufmann.

Pressman, Roger e Maxim, B. (2016). Engenharia de Software-8a Edição. McGraw Hill Brasil.

Samini, Ali e Palmerius, K. L. (2017). Popular performance metrics for evaluation of interaction in virtual and augmented reality. In 2017 International Conference on Cyberworlds $(C W)$, pages 206-209. IEEE.

Santos, M. E. C., Chen, A., Taketomi, T., Yamamoto, G., Miyazaki, J., and Kato, H. (2013). Augmented reality learning experiences: Survey of prototype design and evaluation. IEEE Transactions on learning technologies, 7(1):38-56.

Schulmeyer, Gordon Gordon e McManus, J. I. (1992). Handbook of software quality assurance. Van Nostrand Reinhold Co.

Theden, Philipp e Colsman, H. (2013). Qualitätstechniken: Werkzeuge zur Problemlösung und ständigen Verbesserung. Carl Hanser Verlag GmbH Co KG. 